
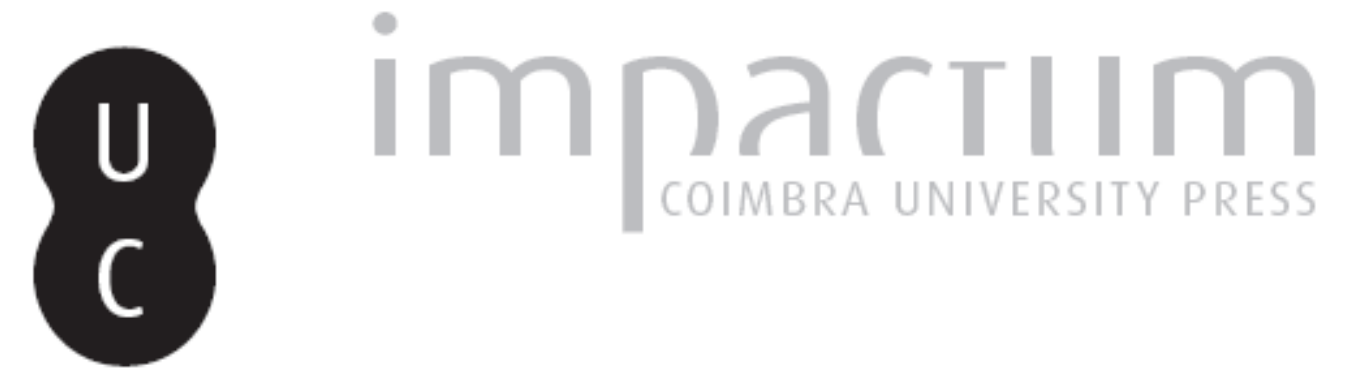

\title{
Rotura traumática de vena cava inferior
}
Autor(es): $\quad$ Lorenzo AE, Hernando; GómezGuillamón F, GarcíaNieto; Anduaga A, Menchaca; Anaya P, Moreno

Publicado por: Imprensa da Universidade de Coimbra

URL persistente:

URI:http://hdl.handle.net/10316.2/33224

DOI:

DOI:http://dx.doi.org/10.14195/1647-8630_24_5

Accessed : $\quad$ 26-Apr-2023 04:18:36

A navegação consulta e descarregamento dos títulos inseridos nas Bibliotecas Digitais UC Digitalis, UC Pombalina e UC Impactum, pressupõem a aceitação plena e sem reservas dos Termos e Condições de Uso destas Bibliotecas Digitais, disponíveis em https://digitalis.uc.pt/pt-pt/termos.

Conforme exposto nos referidos Termos e Condições de Uso, o descarregamento de títulos de acesso restrito requer uma licença válida de autorização devendo o utilizador aceder ao(s) documento(s) a partir de um endereço de IP da instituição detentora da supramencionada licença.

Ao utilizador é apenas permitido o descarregamento para uso pessoal, pelo que o emprego do(s) título(s) descarregado(s) para outro fim, designadamente comercial, carece de autorização do respetivo autor ou editor da obra.

Na medida em que todas as obras da UC Digitalis se encontram protegidas pelo Código do Direito de Autor e Direitos Conexos e demais legislação aplicável, toda a cópia, parcial ou total, deste documento, nos casos em que é legalmente admitida, deverá conter ou fazer-se acompanhar por este aviso.

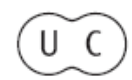


RE VISTA P O R T U G U E S A
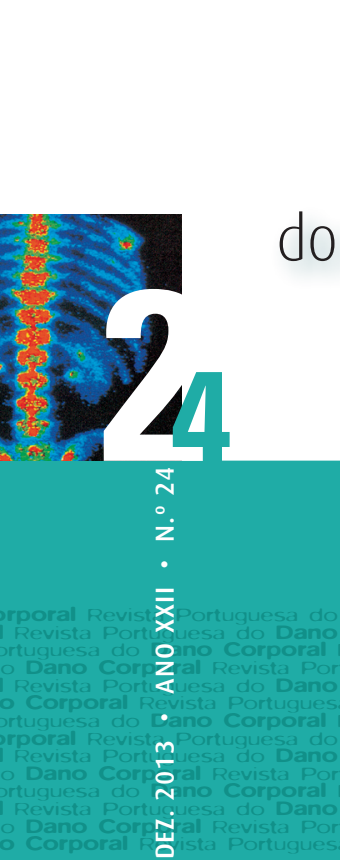

$$
\text { RE VISTA P OR TU/G U E S A }
$$

do

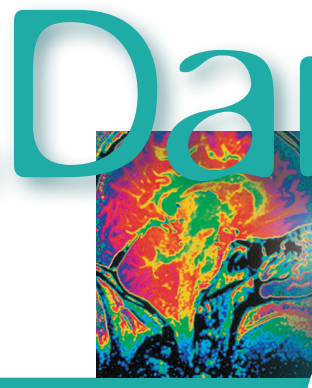

ন

$\stackrel{\circ}{\dot{z}}$

$\dot{\bar{x}}$

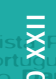

Ono Corporal Revistaral Revista Portuming

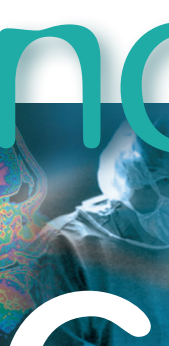

(9)

12

tै. strentes

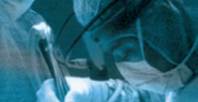

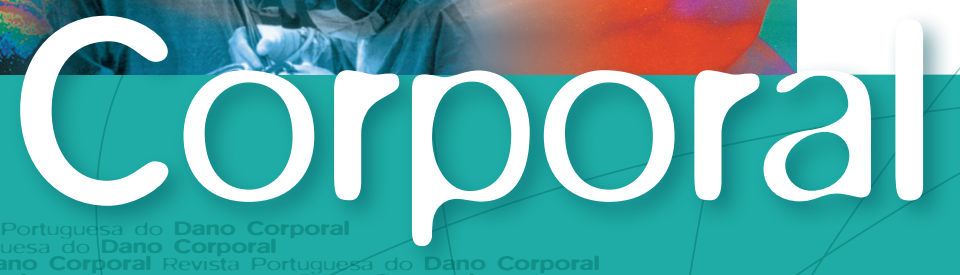

-

m Portuguesa do Dano Corporal Revista Portuguesa do Qano Corporal

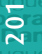
ristal

Corporal Revista Portuquesa do Dano Corpograi Revista Portuquesa do Dang Corporal

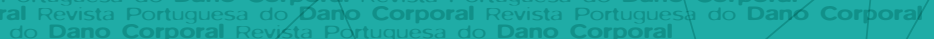

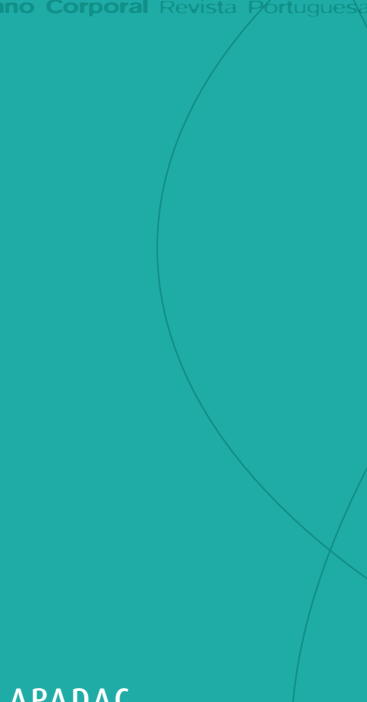

APADAC

ASSOCIAÇÃO PORTUGUESA

DE AVALIAÇÃO

DO DANO CORPORAL

FACULDADE DE MEDICINA

DA UNIVERSIDADE

DE COIMBRA 


\title{
Rotura traumática de vena cava inferior
}

\author{
Hernando Lorenzo AE ${ }^{1}$, García-Nieto Gómez-Guillamón F¹, Menchaca Anduaga $A^{2}$, \\ Moreno Anaya $P^{3}$
}

\section{DESCRIPCIÓN DEL CASO}

Se produce un accidente de tráfico, por impacto lateral sobre el lado derecho de un vehículo utilitario (Seat Ibiza), que circula a velocidad reducida - unos $30 \mathrm{kms} / \mathrm{h}$ - por parte de un camión que circula a una velocidad de unos $80 \mathrm{~km} /$ hora. La ocupante del asiento trasero izquierdo, mujer de 30 años, que hace uso del cinturón de seguridad, con tres puntos de fijación, se encuentra fallecida a la llegada de los servicios médicos de urgencia.

En la autopsia realizada, en la exploración externa del cadáver, se encuentran hematomas en zona abdominal inferior y en hombro izquierdo, compatible con lesiones por cinturón de seguridad, y hematomas en miembros inferiores. En la apertura de cavidades, se encuentra en la cavidad torácica rotura de la $5^{\text {a }}$ costilla izquierda, pulmones exangües y hemotórax masivo derecho de al menos, dos litros de sangre, procedente de un desgarro de unos tres centímetros en la cara lateral derecha de la porción intratorácica de la Vena Cava Inferior (VCI). El pericardio contiene líquido de cantidad y características normales. No se objetivan otros daños macroscópicos en el resto de cavidades.

Esta rotura de Vena Cava Inferior (VCI) produce una hemorragia masiva con resultado de muerte en pocos minutos, siendo cadáver cuando llegan los Servicios médicos de Urgencia.

\footnotetext{
Médico, Servicio de Medicina Intensiva, H. U. "12 de Octubre", Madrid Enfermera, SUMMA 112, Servicio de Urgencias de la Comunidad de Madrid Médico, Servicio de Radiodiagnóstico, H. U. "La Paz". Madrid
} 


\section{DISCUSION Y COMENTARIOS}

Las roturas traumáticas de la vena cava, tanto superior como inferior, son lesiones muy graves y muy poco frecuentes, especialmente las producidas por mecanismo no penetrante, que muchas veces se diagnostican retrospectivamente. Son de mayor gravedad (y con frecuencia mortales), especialmente en aquellos lesionados que llegan a Urgencias en shock y no responden a medidas de reanimación inicial, los que todavía sangran activamente en el momento de la laparotomía y los que tienen heridas en la vena cava retrohepática. De los que llegan a quirófano, la muerte se produce principalmente por exanguinación intraoperatoria. ${ }^{1}$ Se han utilizado varias técnicas de reparación quirúrgica, entre ellas la venorrafia primaria, pero se ha criticado esta técnica debido a su posibilidad de estenosis, trombosis y embolismo.

Carr y otros hicieron un estudio retrospectivo para valorar la morbilidad y pronóstico de este método. Un total de 38 pacientes tratados en el departamento de Cirugía de Trauma del hospital Henry Ford de Detroit, Michigan, EEUU, entre los años 1994 y 1999, tenían lesiones traumáticas en la VCI. Seis murieron en la sala de Urgencias y nueve en el quirófano. De los 38 casos, 3 (el 8\%) se debían a trauma cerrado. La inmensa mayoría de lesiones traumáticas de VCI pueden ser tratadas por compresión directa o pinzado local y venorrafia primaria. Refieren que las reparaciones directas se asocian a unas tasas bajas de complicación por trombosis y embolia ${ }^{2}$.

En el caso de los desgarros de vena cava inferior por traumatismo cerrado externo, son relativamente raros pero muy graves. Van de Wal. y cols., refieren siete casos de laceración de VCI en un periodo de 12 meses. La tercera parte de pacientes no llegan vivos al hospital y, de los que llegan vivos, entre un tercio y la mitad morirán a pesar de una resucitación rápida y una intervención quirúrgica precoz. La alta mortalidad la atribuyen a la dificultad diagnóstica y a los problemas técnicos para su reparación, especialmente en las roturas de la VCI por encima de las venas renales ${ }^{3}$. Refieren el caso de dos lesionados que presentaban desgarro de localización supradiafragmática; como en el caso que presentamos, ambos habían sufrido lesiones de desaceleración y usaban cinturones de seguridad, con una clínica de presentación muy parecida.

En una revisión retrospectiva de los pacientes que son traídos a un centro terciario de trauma a lo largo de siete años, hay dieciséis casos de lesión traumática de VCI, de los que 15 son por mecanismo penetrante y sólo uno por causa no penetrante. Seis de los lesionados murieron. Encuentran que son predictores de muerte el Glasgow Coma Scale (GCS), la necesidad de cirugía para control del sangrado y la necesidad de ligadura de la VCI en el acto quirúrgico ${ }^{4}$. 
Coimbra R, Prado PA. y otros ${ }^{5}$ revisaron retrospectivamente 49 pacientes que sufrieron lesiones de VCI por cualquier causa durante un periodo de 5 años, para detectar los factores relacionados con mortalidad precoz. El mecanismo de lesión más frecuente fue herida por arma de fuego (59\%), seguido por heridas de arma blanca (29\%) y traumatismo cerrado (12\%).

Hubo 4 lesiones en la VCI supradiafragmática, 14 retrohepáticas, 16 suprarrenales y 15 en la porción infrarrenal de la VCI. El hígado fue el órgano lesionado más frecuentemente. La mortalidad fue del 100\% en las lesiones supradiafragmáticas, 71,4\% en las retrohepáticas, 69\% en las suprarrenales y $33 \%$ en las infrarrenales. La rotura de la VCI a nivel diafragmático y la inestabilidad hemodinámica al ingreso fueron la causa más importante de mortalidad precoz. Todos los pacientes con lesión vascular intrabdominal concomitante murieron.

La lesión de la VCI a nivel intratorácico en el caso que describimos se produce a nivel extrapericárdico, produciéndose una hemorragia a pleura; pese a ser la VCI un sistema de baja presión hidrostática, en caso de una rotura de suficiente tamaño, como es en el caso que presentamos, se produce un hemotórax masivo que conduce a la muerte en minutos. En este caso el lesionado usaba cinturón de seguridad y el mecanismo de producción es por desaceleración. La rotura intrapericárdica de vena cava inferior tras una lesión por desaceleración en pacientes que usan cinturón de seguridad, sugiere que cuando el corazón es desplazado hacia delante de forma aguda, el borde rígido del diafragma impedirá que la vena cava inferior siga ese movimiento, produciendo el desgarro de la vena cava inferior en su unión con la aurícula derecha.

Si la laceración de la VCI se produce a nivel intrapericárdico, tendremos un sangrado pericárdico que puede producir un taponamiento cardíaco. El caso posiblemente más frecuente es la laceración del ventrículo derecho y aurícula derechas, que se extiende a la VCI tanto intra como extrapericárdica y puede alcanzar la porción infradiafragmática.

Un mecanismo lesional que se ha propuesto ha sido la lesión por deceleración "en martillo de agua". Couves CM y Heughan $\mathrm{C}^{6}$ refieren el caso de un paciente de 21 años con politraumatismo y que sufrió rotura de vena cava intrapericárdica y que requirió estereotomía para control de la hemorragia. Sugieren la desaceleración "en martillo de agua" como mecanismo lesivo. La vena cava superior está parcialmente fijada por la reflexión del pericardio y su entrada en la aurícula derecha. Con la desaceleración y la inevitable maniobra de Valsalva que acompaña a la lesión, se ha postulado que la vena cava superior revienta por estas fuerzas.

Las lesiones vasculares por desaceleración en el tórax ocurren principalmente a nivel de la aorta, inmediatamente distal a la salida de la arteria subclavia, a nivel 
del ligamento arterioso. Menos frecuentes son las lesiones de otros grandes vasos. Geusens E y otros revisan las causas de ensanchamiento mediastínico radiológico visto en la sala de urgencias después de un trauma torácico no penetrante de alta energía y con grandes fuerzas de desaceleración y refieren la lesión de la aorta y otros grandes vasos intratorácicos, con deterioro hemodinámico habitualmente y ensanchamiento mediastínico en la radiografía de tórax. El diagnóstico se facilita con un TC espiral multicorte ${ }^{7}$. Discuten los signos del ensanchamiento mediastínico, de causas tanto traumáticas como no traumáticas.

Berr I, Bielik E, Killárik V ${ }^{8}$ refieren un lesionado inconsciente, con datos de trauma cerrado torácico y epigástrico, que desarrolló signos de derrame pericárdico y peritoneal, con taponamiento y fallo circulatorio. Se realizó laparotomía y esternotomía medias y se encontró una rotura de unos $5 \mathrm{~cm}$ de longitud en aurícula derecha, pared anterior de la parte intrapericárdica de la vena cava inferior y con una corta extensión a la porción infradiafragmática de la vena cava inferior. Tras control de la hemorragia realizan la reparación vascular con parche de pericardio. El paciente tuvo un postoperatorio complicado siendo dado de alta a los 37 días. A los sietes meses estaba en buena situación sin datos de estenosis vascular en la zona de reparación vascular.

Respecto a la rotura traumática de vena cava, pocos pacientes con rotura de vena cava superior viven lo suficiente para llegar vivos al hospital y poder recibir tratamiento. Ochsner JL, Crawford ES, de Bakey $\mathrm{ME}^{9}$ observaron en una serie de 14 años, 85 pacientes en esta serie, 16 (55\%) murieron antes del ingreso en el hospital; y 39 (45\%) que sobrevivieron para llegar vivos al hospital. De los que tuvieron lesión en vena cava inferior, 37 estaban vivos al ingreso en el hospital, 21 estaban muertos al ingreso. Todos estos pacientes, tenían trauma penetrante, por arma blanca o arma de fuego, y en sólo uno, que llegó vivo al hospital, la lesión se debió a trauma cerrado.

Fey GL, Deren MM, Wesolek JH refieren el caso de un paciente de 43 años que tras un accidente de tráfico presenta hipotensión y taquicardia sin datos externos de traumatismo torácico.

La TC de abdomen no mostró hallazgos, pero por ecografía se diagnosticó taponamiento pericárdico y líquido en el abdomen. Se realizó pericardiocentesis aspirándose sangre no coagulada y laparotomía que mostró sangre intraabdominal y arrancamiento de la cápsula esplénica. Tras esternotomía se vio un desgarro de vena cava inferior que fue reparada ${ }^{10}$.

Peitzman $\mathrm{AB}$ y otros ${ }^{11}$ refieren dos casos de sección intrapericárdica de la VCI, rotura pericárdica y rotura cardiaca. Uno de ellos era conductor de motocicleta y el otro de bicicleta, sufriendo colisión. En los dos casos los servicios sanitarios los encuentran en parada cardiorespiratoria, siendo infructuosa la reanimación. Ambos casos había desgarro pericárdico, desgarro de ventrículo derecho y sección casi completa de la VCI a nivel pericárdico o diafragmático. 
Las medidas de resucitación fueron infructuosas. Hacen una revisión de las lesiones cardiacas por traumatismo cerrado y refieren que la más frecuente es la contusión miocárdica. La rotura cardíaca puede aparecer en traumas más severos y era la causa del 65\% de las muertes después de trauma torácico cerrado. Las causas de muerte son, principalmente, las arritmias, la exanguinación, el taponamiento pericárdico y la insuficiencia cardíaca aguda. Si concomitantemente a la rotura o laceración cardíaca hay una rotura pericárdica que permite el paso de la sangre a la cavidad torácica o el abdomen, se puede producir la muerte por exanguinación. Si la sangre queda contenida en el pericardio, se producirá un taponamiento pericárdico. Es menos frecuente que la muerte se produzca por daño miocárdico extenso e insuficiencia cardíaca aguda ${ }^{12}$.

Parmley LF y cols. revisaron 546 autopsias con lesiones cardíacas no penetrantes y describieron 353 casos de rotura cardiaca en esta serie (el $65 \%)^{13}$. Los autores revisaron 546 pacientes durante un estudio multicéntrico de pacientes politraumatizados. De ellos 7 pacientes $(1,3 \%)$ mostraban desgarro de vena cava inferior, de los cuales 2 tenían desgarro de la cava inferior por encima del diafragma. Ambos pacientes habían sufrido una lesión por desaceleración, y usaban cinturón de seguridad. La rotura intrapericárdica de vena cava inferior tras una lesión por desaceleración en pacientes que usan cinturón de seguridad, sugiere que cuando el corazón es desplazado hacia delante de forma aguda, el borde rígido del diafragma impedirá que la vena cava inferior siga ese movimiento, produciendo el desgarro de la vena cava inferior en su unión con la aurícula derecha.

Tochii M, Sygimura S y cols ${ }^{14}$ refieren dos casos de rotura de VCI, correspondiendo el primero a un paciente de 52 años ingresado tras un accidente de tráfico. El TAC mostró taponamiento cardiaco y hematoma mediastínico. En la cirugía de urgencias se encontró rotura de las aurículas derecha e izquierda a través de la cara caudal del septo interauricular y un desgarro separado de la vena cava inferior intrapericárdica. El segundo caso correspondió a un varón de 35 años que saltó desde un cuarto piso. El TAC mostró rotura de aorta descendente y llevado a cirugía falleció de hemorragia masiva por la rotura aórtica. La exploración reveló rotura de la vena cava inferior intrapericárdica.

Park CS, Wesselhoeft CW Jr ${ }^{15}$ refieren el caso de un niño de 7 años que cayó de la parte de atrás de una moto y sufrió un desgarro de la vena cava inferior intrapericárdica con taponamiento cardiaco. Presentaba hipotensión y distensión abdominal y no había evidencia de traumatismo torácico. La laparotomía mostró un hígado distendido a tensión sin sangre intraabdominal. La estereotomía media mostró un desgarro de vena cava que se reparó. Refieren que la distensión abdominal creciente con un agrandamiento hepático sugiere desgarro traumático de vena cava suprahepática. 
Seoudi HM, Lefrak EA y cols. refieren un caso de supervivencia en un joven de 19 años tras un traumatismo cerrado por accidente de tráfico, con rotura de vena cava inferior intrapericárdica, rotura de aorta y rotura de bazo ${ }^{16}$.

Respecto a la rotura de vena cava inferior, el traumatismo cerrado de vena cava inferior (VCI) es infrecuente y se produce en el 1\% al 10\% de los pacientes con traumatismo cerrado. Las lesiones generalmente se deben a desaceleración, que produce rotura atriocava o desgarro de las venas hepáticas y a veces se complica con hemorragia incontrolable ${ }^{17}$.

Klein SR y cols. revisaron 38 pacientes tratados desde 1983 a 1990 en el Centro Médico de Harbor-UCLA, California, EEUU. 14 de las lesiones se debían a traumatismo cerrado. 30 de los 38 (79\%) sobrevivieron. La lesión de cava era retrohepática en 12 (en 3 afectaba a las venas hepáticas), suprarrenal en 7 , pararrenal en 9 e infrarrenal en $10^{18}$.

Entre los predictores de la mortalidad y el manejo de los pacientes con lesiones traumáticas de VCI (Huerta S y cols. "Predictors of mortality and management of patients with traumatic inferior vena cava injuries", Am Surg, 2006 Apr; 72(4): 290-296), los autores revisaron retrospectivamente todos los pacientes de trauma con lesiones de VCI durante un periodo de 7 años. Identificaron 36 lesiones de VCI con una mortalidad del 56\%, siendo el mecanismo de lesión traumatismo cerrado en el $28 \%$ y penetrante en el $72 \%$. No hubo diferencia en la mortalidad en base al mecanismo de lesión.

Las lesiones con mayor proximidad al corazón se asociaron a mayor mortalidad. Los que no sobrevivieron tenían una puntuación más alta en la escala de gravedad de lesión (ISS), una presión arterial sistólica más baja y una puntuación más baja en la escala de Glasgow en Urgencias.

Graham CA y otros refieren que el desgarro de vena cava superior es un hallazgo poco frecuente del traumatismo cerrado y es casi invariablemente mortal, se presenta un caso de accidente de tráfico a alta velocidad y se discuten los factores relacionados con la supervivencia ${ }^{19}$.

Recientemente se ha descrito el uso de endoprótesis para reparación de lesión de $\mathrm{VCI}$ en un paciente que se consideró que presentaba un riesgo muy elevado para la reparación quirúrgica ${ }^{20}$.

Dada la alta mortalidad de las lesiones traumáticas de las venas retrohepáticas por la dificultad técnica para conseguir una adecuada hemostasia, se han propuesto múltiples estrategias de tratamiento. Kaoutzanis $\mathrm{Cy}$ otros refieren un caso de sección traumática de las dos principales venas hepáticas extraparenquimatosas y laceración de la VCI retrohepática, como lesiones aisladas después de un trauma abdominal cerrado, reparadas con éxito usando bypass cardiopulmonar y parada circulatoria hipodérmica ${ }^{21}$. 


\section{Bibliografía}

${ }^{1}$ Buckman RF, Pathak AS, Badellino MM, et al. Injuries of the inferior vena cava. Surg Clin North Am. 2001;81:1431-47.

${ }^{2}$ Carr JA, Kralovich KA, Patton JH, Horst HM. Primary venorraphy for traumatic inferior vena cava injuries. Am Surg. 2001 Mar; 67(3):207-13; discusión 213-214).

${ }^{3}$ Van de Wal H.J.C.M., Draaisma J.M. y cols. Rupture of the supradiaphragmatic inferior vena cava by blunt decelerating trauma: case report, J. Trauma. 1990 Jan 32(1): 111-3.

${ }^{4}$ Cudworth M, Fulle A, Ramos JP, Arriagada I. GCS as a predictor of mortality in patients with traumatic inferior vena cava injuries: a retrospective review of 16 cases. World J Emerg Surg. 2013 Dec 29;8(1):59. doi: 10.1186/1749-7922-8-59.

${ }^{5}$ Coimbra R, Prado PA, Araujo LH, Candelaria PA, Caffaro RA, Rasslam S. Factors related to mortality in inferior vena cava injuries. A 5 year experience. Int Surg, 1994 AprJun;79(2): 138-41.

${ }^{6}$ Couves CM, Heughan C. Laceration of superior vena cava due to blunt trauma: successful surgical management. Can. J. Surg 1981, 24(4): 402-3.

${ }^{7}$ Geusens E, Pans S, Prinsloo J, Fourneau I. The widened mediastinum in trauma patients Eur. J Emerg Med 2005, Aug 12(4): 179-84.

${ }^{8}$ Berr I, Bielik E, Killárik V., "Intrapericardial rupture of the inferior vena cava caused by blunt trauma”, Rozhl Chir, 2002 Aug: 81 (8): 398-400 (Abstract en ingles).

${ }^{9}$ Ochsner JL, Crawford ES, de Bakey ME, Injuries of the vena cava caused by external trauma Surgery, 1961; 49: 397-405.

${ }^{10}$ Fey GL, Deren MM, Wesolek JH, Intrapericardial caval injury due to blunt trauma. Conn Med. 1999 May: 63 (5): 259-60.

${ }^{11}$ Peitzman AB, Udekwu AO, Pevec W, Albrink M. Transection of the inferior vena cava from blunt thoracic trauma: case reports. J. Trauma. 1989 Apr;29(4): 534-6.

12 Dow RW. Myocardial rupture caused by trauma. Surgery. 1982 Feb;91(2):246-7.

${ }^{13}$ Parmley LF, Manion WC, Mattingly TW. Nonpenetrating traumatic injury of the heart. Circulation. 1958 Sep;18(3):371-96.

${ }^{14}$ Tochii M, Sygimura S. y cols. Blunt rupture of the intrapericardial inferior vena cava: report of two cases, Kyobu Geka 2000 Feb. 53 (2): 145-7 (abstract en ingles).

${ }^{15}$ Park CS, Wesselhoeft CW Jr. "Blunt traumatic laceration of the suprahepatic inferior vena cava presenting as abdominal pain and shock in a child: a case report". Trauma. 1995 Jan. 38 (1): 68-9.

${ }^{16}$ Seoudi HM1, Lefrak EA, Speir AM, Hendershot K, Moynihan J, Fakhry SM.

Survival following combined intrapericardial inferior vena cava and thoracic aortic injury caused by blunt trauma. J Trauma. 2008 Jul; 65 (1): 218-21.

${ }^{17}$ Cole K, Shadis R, Sullivan TR Jr. Retrohepatic hematoma causing caval compression after blunt abdominal trauma. J Surg Educ. 2009 Jan-Feb; 66(1): 48-50.

${ }^{18}$ Klein SR, Baumgartner FJ, Bongard FS. Contemporary management strategy for major inferior vena caval injuries. J Trauma. 1994 Jul;37(1):35-41; discussion 41-2.

${ }^{19}$ Graham CA, McLeod LS, Mitchell RG, Wyatt JP, Walker WS. Survival after laceration of the superior vena cava from blunt chest trauma. Eur J Emerg Med. 1996 Sep; 3(3):191-3. 
${ }^{20}$ Sam AD, Frusha JD, McNeil JW, Olinde AJ. Repair of a blunt traumatic inferior vena cava laceration with commercially available endografts. J Vasc Surg. 2006 Apr;43(4):841-3.

${ }^{21}$ Kaoutzanis C, Evangelakis E, Kokkinos C, Kaoutzanis G. Successful repair of injured hepatic veins and inferior vena cava following blunt traumatic injury, by using cardiopulmonary bypass and hypothermic circulatory arrest. Interact Cardiovasc Thorac Surg. 2011 Jan;12(1):84-6.

Resumo: Rotura traumática de veia cava inferior

Trata-se de um caso de rotura traumática da Veia Cava Inferior (VCI) a nivel intratorácico e extrapericárdico, que deu origem a um volumoso hemotórax, documentado na autópsia, numa mulher jovem com cinto de segurança e ocupante do banco traseiro esquerdo, após acidente de viação com choque lateral no lado oposto (lado direito), de que resultou morte imediata. Comenta-se a bibliografia consultada.

Palavras-chave: Rotura traumática; veia cava inferior intratorácica; choque lateral direito.

Abstract: Traumatic rupture of the inferior vena cava

A case of rupture of supradiaphragmatic extracardiac inferior vena cava is described. It happened as a consequence of blunt trauma after a front to right-side crash between a truck and a car. The passenger, a three point belted 30 year old woman, was seated in the left rear seat. She died at the accident site. Bibliography related to the topic is commented.

Keywords: Traumatic rupture; intrathoracic inferior vena cava; blunt trauma; far-side crash.

\section{Pedido de separatas:}

ANTONIO E. HERNANDO LORENZO

herloren@arrakis.es 